\title{
Implication of diethylcarbamazine induced morbidity and the role of cellular responses associated with bancroftian filariasis pathologies
}

\author{
W.H. MAKUNDE ${ }^{1 *}$, M.L. KAMUGISHA ${ }^{1}$, R.A. MAKUNDE ${ }^{2}$, M.N. MALECELA-LAZARO ${ }^{3}$ and A.Y. KITUA ${ }^{3}$ \\ ${ }^{1}$ National Institute for Medical Research, Tanga Research Centre, P.O. Box 950, Tanga, Tanzania; ${ }^{2}$ Bombo Regional \\ Hospital, Tanga, Tanzania; ${ }^{3}$ National Institute for Medical Research Headquarters, \\ Dar es Salaam, Tanzania
}

\begin{abstract}
Pre and post-diethylcarbamazine treatment clinical expression, microfilaraemia prevalence and cellular responses were investigated in individuals in Tanga, Tanzania. Fifty-seven male individuals (aged =15 years old) were identified for further studies on IL-4, IL-6, IL-8, IFN- $\gamma$, IL- $\beta$, TNF- $\alpha$ and nitric oxide in plasma and hydrocoele fluid. Microfilarial prevalence in the examined individuals was $12 \%$ with a geometric mean intensity (GMI) of $838 \mathrm{mff} / \mathrm{ml}$ in a community with a population of 1018 individuals. Microfilaraemic hydrocoele stage II and III were the most frequent pathologies observed with prevalence of $17.5 \%$ and $42.1 \%$, respectively. All study individuals treated with diethylcarbamazine (DEC) standard dose of $6 \mathrm{mg} / \mathrm{kg}$ experienced post-treatment adverse events. There was no direct relationship between elevated IL6 and the occurrence and severity of clinical adverse effects post-treatment. The findings from this study suggests that, blood elevated cytokine profile is not the main etiological factor in the inflammatory responses developing after treatment of bancroftian filariasis infections and pathology with DEC. Plasma levels of cellular (cytokines) responses during treatment revealed a proportion of symptomatic patients. Prior to treatment, patients with hydroecoele had high levels of IL-6 than those without the pathology. In conclusion these findings do not support the hypothesis that pro-inflammatory cytokines are directly responsible for adverse events to DEC chemotherapy in bancroftian filariasis infections and pathologies such as hydrocoele, lymphoedema and elephantiasis.
\end{abstract}

\section{Introduction}

Lymphatic filariasis (LF) remains a major cause of morbidity and social-psychological problems to patients and relatives in the tropical and subtropical regions throughout the world (Evans et al., 1993). The recent development of new strategies for communitydirected chemotherapy provides hope for the longterm control of LF in many endemic areas (Ottesen $e t$ al., 1997). Current chemotherapy has little impact on the resolution of chronic pathology and emphasizes the need for a clearer understanding of the pathogenesis of filarial pathology, subsequently coming up with a feasible sustainable strategy for morbidity control at individual and community level.

Pathological conditions that are associated with variety of clinical and sub-clinical syndromes are linked with inflammatory responses/reactions. Recently it has been shown that, the responses are triggered by the Wolbachia surface protein (WSP) when getting access into the general circulation due to natural death of adult parasite or through drugs with anti-filarial activity. Clinically the reactions are expressed as acute filarial lymphangioadenitis (AFL) and acute dermatolymphangioadenitis (ADLA). These conditions are characterised by recurrent attacks of inflammation of lymph nodes and vessels often accompanied by systemic febrile responses. In common with other febrile conditions, the severity of
ADLA has been correlated with increasing response to serum levels of TNF-a (Das et al; 1996). Similar symptoms are experienced during adverse reactions to chemotherapy, and are associated with systemic increases of IL-Ib, IL-6, IFN $\gamma$ or TNF- $\alpha$ (Yazdanbakhsh et al., 1992; Turner et al., 1994). However, constant repeated inflammatory responses when not arrested or inhibited promptly, will progress to chronic clinical expressions such as hydrocoele and lymphoedema which when precipitated might lead to elephantiasis, dermatolymphangioadenitis (DLA) and tropical pulmonary filarial eosinophilia (TPFE). On the other hand a number of studies have shown that, antigen-specific inflammatory T-cell mediated responses are associated with some of these conditions (Lal \& Ottesen, 1988) although the role of some of these inflammatory mediators is poorly understood.

The diversity of filarial pathology may partly reflect the variety of aetiological hypothesis speculated to contribute to the pathogenesis of the disease. Such hypotheses include the parasite, the immune response, vectors and secondary opportunistic infections (Maizels \& Denham, 1992; Dreyer et al., 1994; Shenoy et al., 1995; Olszewski et al., 1997). Of recent, Wolbachia endobacteria of many filarial nematodes, have been implicated in the pathogenesis of filarial diseases, possibly through their endotoxin and surface protein. It is also thought that, inflammatory adverse events are a signal of rapid destruction of filarial parasite that releases bacterial symbionts into the blood 
and tissue (Cross et al., 2001; Keiser et al., 2002). Studies elsewhere have shown clearly that there is a correlation of IL-6 and intensity of infection and development of fever (Turner et al., 1994). Some studies have shown this type of responses post-antifilarial treatment. However, it is not clear whether such responses are entirely from a unique infection or treatment result of Wolbachia dying natural death or multiple co-existing infections releasing LP-like substances causing cytokines triggering elevation of cellular responses. It is therefore crucial to continue to refine these observations for a better understanding.

Pro-inflammatory cytokines have been shown to play an early role in parasitic infections by influencing the course of the infection directly and by regulating the specific immune response (Titus et al., 1991). These cytokines also orchestrate a variety of effect mechanisms under the control of specific immune responses (Titus et al., 1991, Stadnyk \& Gauldie, 1991). Whatever the underlying cause of inflammation, mechanisms leading to the development, maintenance and exacerbation of pathology may share common key components. It has already been observed in several clinical conditions and experimental models that the induction and regulation of inflammation is under the control of pro-inflammatory cytokines including ILIb, IL-6, IL-8, IFN $\gamma$, TNF and nitric oxide. Interleukine-1, IL-6 and TNF display a wide-range of immuno-stimulatory functions including induction of fever, reactive nitrogen and oxygen intermediates and the production of acute phase proteins (Titus et al., 1991; Stadnyk \& Gauldie, 1991). Although a variety of functions are common to these cytokines, it was confirmed that in IL-6 gene-targeted mice, the optimal responses to trauma and infection are dependent on IL-6 in vivo (Kopf et al., 1994; Fattori et al ., 1994). In this study we have carried out an analysis of the pro-inflammatory cytokine responses in plasma and hydrocoele fluid associated with different clinical syndromes and accompanying adverse events to DEC chemotherapy in Tanzanians infected with Wuchereria bancrofti. An understanding of the nature of these inflammatory processes may provide new insight into the pathogenesis of lymphatic filariasis and the development of appropriate strategies for its clinical management.

\section{Materials and Methods}

\section{Study area and population}

The study was conducted at Mwarongo-Mwindura village in Tanga City in north-east Tanzania where bancroftian filariasis is endemic. The survey was carried out between 1999 and 2001. The study area experiences short rains from October-November and long rains from
March-June. The average annual rainfall is about 1300 $\mathrm{mm}$ and the mean monthly temperatures range from $20^{\circ} \mathrm{C}$ in July to $32^{\circ} \mathrm{C}$ in February. The relative humidity at noon varies from $65 \%$ in July to near $100 \%$ in March. The village is adjacent to mangrove swamps and surrounded by low shrub and grassland. The main economic activity in the area is fishing and small-scale farming of coconut, cassava, rice and mango.

Before starting the survey, village meetings were held and the villagers were visited to get a better impression of the respective community and to slowly acquire knowledge about the village and create a relationship with the villagers. The project team made a house-to-house visit collecting information on name, sex, age and length of residence against each subject. The census covered all villagers both males and females aged $\geq 15$ years. Males aged $\geq 15$ years with bancroftian filariasis were selected to participate in this study after clinical and parasitological examination. A verbal consent was obtained from each individual who volunteered to participate in the study.

\section{Clinical and parasitological examination}

Fifty-seven individuals were identified for the study and their inclusion criteria for the recruitment was based on the following: (i) microfilaraemia $100 \mathrm{mff} / \mathrm{ml}$ no hydrocoele (Group 1, N=19), (ii) hydrocoele stage II and microfilaraemia 100mff/ml (Group 2, N=13) and (iii) hydrocoele stage II and no microfilaraemia (Group $3, \mathrm{~N}=25$ ).

Pre-treatment detailed clinical history and physical examination of the identified individuals was conducted. Study individuals were examined in the village dispensary behind a screen to maintain privacy. They were asked whether hydrocoelectomy had been conducted in the previous years or not. Addition to this examination of the genitalia for bancroftian filariasis, clinical disease for the signs of the condition was also conducted and graded (McMahon et al., 1981; Meyrowitsch et al., 1995).

Blood pressure was measured pre-, during and post-DEC administration in three positions; sitting, standing upright and lying supine. The mean systolicdiastolic blood pressure of the three readings was recorded. Body temperature was taken using a conventional clinical thermometer placed within the axilla for 3 minutes, before, $6-8$ hours post-DEC and at day 13 post-DEC exposures.

Blood specimen for microfilariae (mff) was carried out between $21.00 \mathrm{~h}$ and $01.00 \mathrm{~h}$. A $100 \mu \mathrm{l}$ of finger prick blood specimen was collected in heparinized capillary tubes to identify microfilaria-positive individuals for further studies on cellular responses. Thereafter samples were immediately transferred into $1 \mathrm{ml}$ of $3 \%$ acetic acid. At the laboratory, the microfilariae in the 
specimens were quantified by using the counting chamber technique (McMahon et al., 1979), and the number of $\mathrm{mff} / \mathrm{ml}$ was calculated.

\section{Plasma cytokines determination}

Venous blood was collected from the 57-study individuals using $10 \mathrm{ml}$ EDTA vacutainer tubes. The blood specimens were collected before and after DEC administration and marked sample 0, 6-8 hours (sample 1) and on day 13 (sample 2), respectively. DEC was administered at $6 \mathrm{mg} / \mathrm{kg}$ in a single dose. The development of adverse reactions was monitored by direct observation and questioning of the patients thirty minutes after DEC on the day of treatment and the following day. Adverse reactions were categorized into systemic (generalized) and localized.

Blood specimens were kept inside a cooler box with ice for no longer than 3 hours before being centrifuged at 1,500 rpm for 3 minutes. The plasma was transferred into pyrogen free plastic tubes by using plastic Pasteur pipettes and stored at $-25^{\circ} \mathrm{C}$ until transported on dry ice to Liverpool where they were stored at $-70^{\circ} \mathrm{C}$ until analysed.

Levels of plasma interleukin-6 (IL-6) were assayed using a commercially available ELISA (Quantikine Human IL-6 Immunoassay, Catalogue

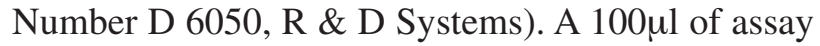
diluent RDIA was added to each of the 96 well plates, then $150 \mu$ l of sample per well was added, and covered by adhesive strip provided by the kit, and incubated for 2 hours at room temperature. After this, each well was aspirated and washed with Wash Buffer $(400 \mu \mathrm{l})$ three times for a total of four washes. Thereafter 200 $\mu l$ of IL- 6 conjugate was added to each well and covered with a new adhesive strip and incubated for 2 hours at room temperature. Then $200 \mu l$ of substrate solution was added to each well, and incubated for 20 minutes at room temperature. After this, $50 \mu l$ of stop solution was added to each well, and then a uniform colour change was observed. The optical density of each well was determined within 30 minutes using a spectrophotometer set at $470 \mathrm{~nm}$ wavelength.
A flask of confluent WEHI 164 SC 14 cells was treated with trypsin. The flask was incubated for 20 minutes until the cells started to float off the bottom. Thereafter the cells were transferred into $10 \mathrm{ml}$ of Roswell Park Memorial Institute Medium in a plastic universal tube. Then $10 \%$ foetal calf serum was added to the flask, and cells were centrifuged for 5 minutes at $1000 \mathrm{rpm}$. The pellet was re-suspended in $10 \mathrm{ml}$ media, and centrifuged again for $5 \mathrm{~min}$. Finally the cells were counted to give a concentration of $3 \times 10^{5} /$ $\mathrm{ml}$.

$100 \mathrm{ul}$ cells $(3 \mathrm{x} 104)$ were transferred into 96well plates. The cells were incubated for 5 hours to allow adherence to the plate. Into each well $1 \mathrm{ng} / \mathrm{ml}$ Penicillin-Streptomycin was added. The TNF standards were serially diluted and added as $50 \mu \mathrm{l}$ of Penicillin-Streptomycin + TNF. Standards and samples were incubated at $37^{\circ} \mathrm{C}$ in $5 \% \mathrm{CO}^{2}$ overnight. After this, the media was poured off the wells and blotted on tissue. The cells were then stained with $25 \mu$ l of $0.5 \%$ ethyl-violet in $20 \%$ methanol for approximately $1 \mathrm{~min}$ and washed gently for $1 \mathrm{~min}$ with PBS. The cells were solubilised with $1 \%$ Lauryl sulfate and 50 $\mathrm{ml}$ per well was used to lyse the cells. The plates were read at $630 \mathrm{~nm}$ wavelength.

\section{Results}

\section{Parasitological and clinical status}

A total of 1018 (females $=541$; males $=477)$ aged $\geq 15$ years old were examined both clinically and parasitologically. The prevalence of Wuchereria bancrofti infection in this population was $12 \%(120 / 1018)(95 \%$ $\mathrm{CI}=9.9-13.9 \%)$. The mff intensity among positive individuals ranged from $10-4510 \mathrm{mff} / \mathrm{ml}$ with a geometric mean intensity (GMI) of $838 \mathrm{mff} / \mathrm{ml}$. The mean for individuals in group 1 was 3598 (95\% CI=1578$8204)$ and for those in group 2 was 1503 (95\% CI $=610-3706)$. Thirteen percent $(69 / 477)$ of the males presented this condition, with chyluria been observed in one individual (54 years old). Lymphoedema and limb elephantiasis were not common.

Table 1: Type of adverse events experienced after oral single dose of $6 \mathrm{mg} / \mathrm{kg}$ DEC administered to individuals with $\boldsymbol{W}$. bancrofti microfilaraemia and hydrocoele

\begin{tabular}{lccc}
\hline Systemic adverse events & Local adverse events & $\begin{array}{c}\text { Proportion \% of } \\
\text { adeverse events }\end{array}$ \\
\hline Type of reaction & $\begin{array}{c}\text { Proportion (\%) of } \\
\text { adverse events }\end{array}$ & Type of reaction & 33.3 \\
\hline Nausea & 19.0 & Orchitis & 22.7 \\
General malaise & 19.0 & Funiculitis & 19.7 \\
Dizziness & 17.5 & Lymphadenitis & 15.2 \\
Fever & 17.5 & Epididymo-orchitis & 7.6 \\
Vomiting & 15.9 & Itching & 1.5 \\
Headache & 11.1 & Transient lymphoedema & \\
\hline
\end{tabular}




\section{Clinical disease and adverse events}

In the 57 studied individuals, 19 presented with microfilaraemia without hydrocoele and 25 had hydrocoele without microfilaraemia. Hydrocoele was the commonest clinical feature observed in this village. Thirteen individuals were observed to present hydrocoele stages II-IV. The mean length of time since the individuals felt that the testicle was swollen till the examination day was 5 years (range $=1-8$ years). Fiftysix percent (32/57) of the individuals with hydrocoele, their disease stage ranged between stage II and IV, with stages II and III being most frequent. Hydrocoele stage I was not observed. Hydrocoele was observed among individuals $\geq 20$ years old. Hydrocoele stages II and III were common in both younger and old males although stage III was more common in older males. Acute forms of the disease (AFL, ADLA, orchitis funiculitis and epididymitis) were not observed during the survey period in the village.

\section{Adverse events following DEC administration}

All study individuals experienced adverse events 2-3 hours following oral DEC administration. Majority of these events were reported between 6-8 hours postDEC and lasted for up to 3 days in all individuals except for two. Events reported were mild and moderate in terms of severity and were categorized into systemic and local (Table 1).

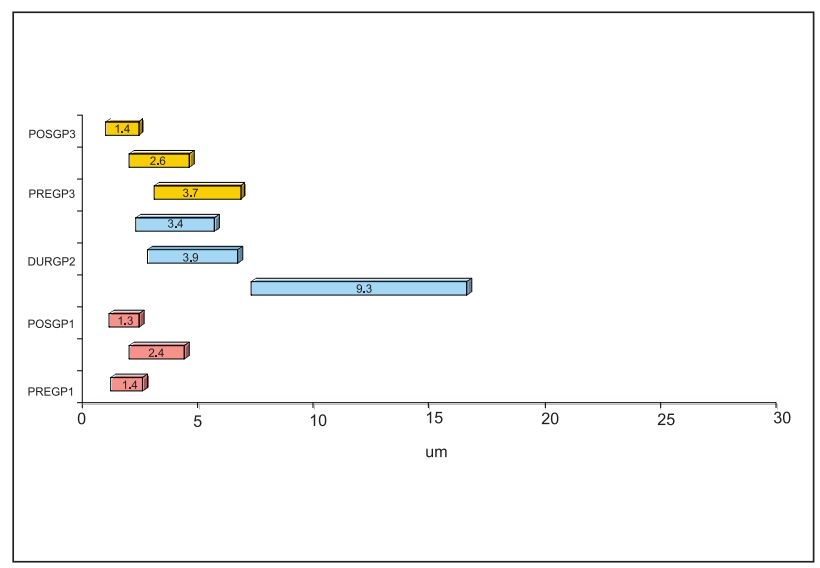

Figure 1: Geometric mean with 95\% CI of plasma levels of IL6 of individuals before, during and after DEC administration

\section{Plasma levels of IL-6}

Twenty-one percent of individuals with microfilariae but no hydrocoele had a slight elevation of IL-6 pre-DEC phase (Figure 1). Those individuals with the pathology (38\%) had significantly higher levels of IL-6 prior to treatment. Individuals without the pathology (58\%) did not show any IL-6 response throughout the entire study period despite suffering adverse reactions to DEC treatment. Twenty-six percent (5/19) presented with moderate increase of IL-6 plasma levels between 6-8 hours post-DEC treatment and with the plasma levels gradually normalising at day 13 post-DEC treatment. In Groups 2 and 3 individuals with pathology, 13\% demonstrated a moderate increase of IL- 6 during treatment. However, 26\% (10/38) of the individuals with hydrocoele did not demonstrate any IL-6 responses in any of the plasma specimens. Twelve (32\%) of those patients with hydrocoele demonstrated a decrease in IL-6 levels between 6 and 8 hours following DEC treatment.

\section{TNF- $\alpha$ in plasma and hydrocoele fluid}

In patients without pathology, 12/19 (63\%) (Group 1) presented an increase in TNF- $\alpha$ levels $6-8$ hours postDEC treatment. Forty-two percent (5/12) of the individuals demonstrated an increase in TNF- $\alpha$ 6-8 hours after DEC treatment, whereas 50\% (6/12) showed a continued increase in plasma TNF- $\alpha$ levels 13 days after DEC treatment (Figure 2). Those patients with hydrocoele presented with a variety of pattern of TNF- $\alpha$ responses at individual level, although there was increase, decrease and no change in levels of TNF- $\alpha$ in their plasma specimens.

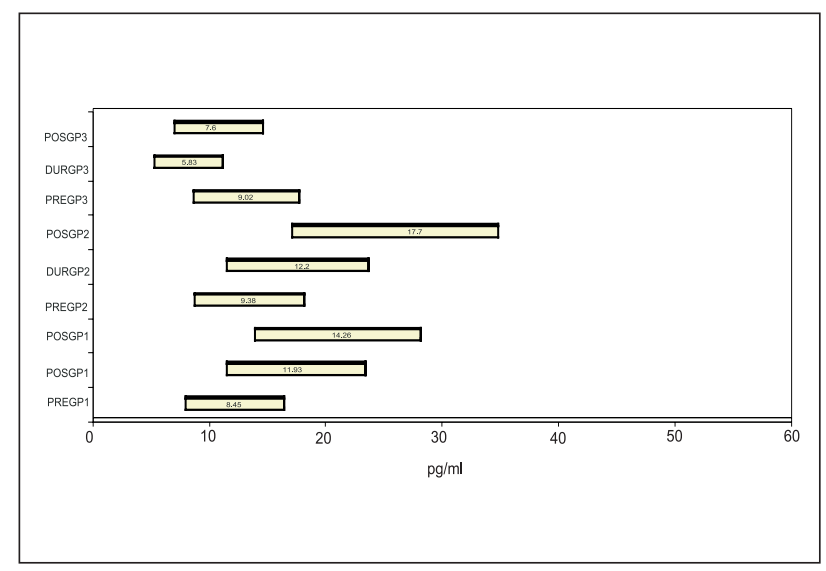

Figure 2: Geometric mean with $95 \% \mathrm{CI}$ of plasma levels of TFN- $\alpha$ in the study population before, during and after DEC administration

\section{Discussion}

The prevalence of microfilariae in the examined population/individuals was slightly lower $(12 \%)$ as compared to previous studies that showed to range from $15-26 \%$. The East African coast is highly endemic for bancroftian filariasis, but with some variation in prevalence, intensity and severity occurring from one place to another (Wegesa et al., 1979; Abaru et al., 1980; McMahon et al., 1981). As in other regions of East Africa, hydrocoele is a common chronic clinical form of the disease in this community. This provides the opportunity to compare the pro-inflammatory cytokine response during treatment between patients with and without the pathology. A prevalence of $13 \%$ 
in males aged $\geq 15$ years that was observed in this study is lower than $15-26 \%$ that was reported previously in the coastal areas of Tanzania (Abaru et al., 1980; Wegesa et al., 1979; McMahon et al., 1981).

The early cases of hydrocoele were recorded in young adult males with a prevalence of $50 \%$. Large hydrocoeles at stage II and III have been reported to be a burden to the affected males causing difficulties in walking, working and sexual activity (Muhondwa, 1978). Although our study showed lack of a correlation between large hydrocoeles and blood pressure, such a phenomenon has been previously reported by Jordan (1957). Microfilaraemia was common in males with hydrocoeles. Similar observations have been reported by several studies elsewhere in Tanzania (Wegesa et al., 1979; Abaru et al., 1980; Makunde, 1995; Meyrowitsch et al., 1995). Small hydrocoeles (stages I) was not recorded probably because of exclusion of the age $<15$ years old.

Previous studies on the role of pro-inflammatory cytokines in the adverse events to DEC have been carried out on small numbers of asymptomatic microfilaraemic patients. These studies showed moderate increases of IL- 6 and TNF correlated with the presence of adverse events (Yazdankakhsh et al., 1992; Turner et al., 1994). In particular, IL-6 levels correlated with the development of fever and in onchocerciasis associated with levels of microfilardemia (Turner et al., 1994). The results of the present study show that a proportion of asymptomatic microfilaraemic patients do show a moderate IL- 6 and TNF responses despite experiencing adverse reactions to DEC treatment.

A major difference between patients with pathology and those without pathology was observed in pre-chemotherapy levels of IL-6. High levels of IL-6 were present in about $40 \%$ of those patients with hydrocoele (data not shown). One possible explanation for this observation may be the association of secondary responses. Alternatively, the development of filarial pathology is likely to be associated with pro-inflammatory responses. Wolbachia is also likely to contribute to these inflammatory phenomena through its surface protein (WSP), independently from its endotoxin component. Similarly, recent observation has shown that, antibody reactivity to WSP does correlate with the presentation of lymphoedema and hydrocoele pathology in subjects infected with $W$. bancrofti, indicating that immune responses to these endobacteria might trigger chronic morbidity (Punkosdy et al., 2003).

It is therefore concluded that, a proportion of asymptomatic microfilaraemic individuals do present pro-inflammatory cytokine response following DEC treatment. However, this is not directly related to adverse event, as a high number of individuals experiencing adverse events did not show a cytokine response in our study. Individuals with hydrocoele in our study presented high levels of IL-6 prior DEC treatment. In addition, the observations that individuals with hydrocoele had high levels of Il-6, IL-8, TNF- $\alpha$ and T-cell mediated and that pro-inflammatory reactions are not responsible for the adverse events following treatment with DEC.

\section{Acknowledgments}

The authors are very grateful to Prof. Allen Shankle who provided space to run the TNF and IL-6 assays. Dr. Mtumwa Mwako, the District Medical Officer for Pangani is thanked for facilitating the field activities. Mr. Charles Guzo is thanked for his excellent field assistance.

\section{References}

Abaru, D.E., McMahon, J.E., Marshall, T.F. de C., Hamilton, P.J.S., Vaughan, J.P. \& Wegesa, P. (1980) Tanzania Filariasis Project. Studies on microfilaraemia and selected clinical manifestations of Bancroftian filariasis. Acta Tropica 37, 63-71.

Cross, H.F., Haarbrink, M., Egerton, G., Yazdanbakhsh, M. \& Taylor, M.J. (2001) Severe reactions to filarial chemotherapy and release of Wolbachia endosymbionts into blood. Lancet 358, 18731875.

Das, B.K., Sahoo, P.K. \& Ravindran, B. (1996) A role for tumour necrosis factor-alpha in acute lymphatic filariasis. Parasite Immunology $\mathbf{1 8}$, 421-423.

Dreyer, G., Pires, M.L., Andrade, L.D., Lopes, E., Medeiros, Z., Tenorio, J., Coutinho, A. \& Norões, J. (1994) Tolerance of diethylcarbamazine by microfilaraemic and amicrofilaraemic individuals in an endemic area of bancroftian filaraiasis, Recife, Brazil. Transactions of the Royal Society of Tropical Medicine and Hygiene 88, 232-236.

Evans, B.D., Gelband, H. \& Vlassoff, C. (1993) Social and economical factors and the control of lymphatic filariasis: a review. Acta Tropica $\mathbf{5 4}$, $1-26$.

Fattori, E., Cappelletti, M., Costa, P., Sellitte, C., Cantoni, L., Carelli, M., Faggioni, R., Fantuzzi, G., Ghezzi, P. \& Poli, V. (1994) Defective inflammatory response in interleukin-6 deficient mice. Journal of Experimental Medicine 180, 1243-1250.

Jordan, P. (1957) The fevers of Africa; 5. Clinical bancroftian filariasis in Tanganyika. Central African Journal of Medicine 3, 18-23. 
Keiser, P.B., Reynolds, S.M., Awadzi, K., Ottesen, A.E., Taylor, M.J. \& Nutman, B.T. (2002) Bacteria endosymbionts of Onchocerca volvulus in the pathogenesis of post treatment reactions. Journal of Infectious Diseases 185, 805-811.

Kopf, M., Baumann, H., Freer, G., Freudenberg, M., Lamers, M., Kishimoto, T., Zinkernagel, R., Bleuthmann, H. \& Kohler, G. (1994) Impaired immune and acute-phase responses in interleukine- 6 deficient mice. Nature 368, 339-342.

Lal, R.B. \& Ottesen, E.A. (1988) Enhanced diagnostic specificity in human filariasis by $\mathrm{IgG} 4$ antibody assessment. Journal of Infectious Diseases 158, 1034-1037.

Maizels, R.M. \& Denham, D.A. (1992) D i e th y 1 c a r b a m a z i n e : immunopharmacological interaction of an anti-filarial drug. Parasitology 105, S49-S60.

Makunde, W.H. (1995) The Role of Pro-Inflammatory Cytokines and Nitric Oxide from Plasma and Hydrocoele Fluid in the Adverse Reaction to Diethylcarbamazine (DEC) in Bancroftian Filariasis in Tanzania. MTropMed. Dissertation. University of Liverpool, UK.

McMahon, J.E., Marshall, T.F. de C., Vaughan, J.P. \& Abaru, D.E. (1979) Bancroftian filariasis: a comparison of microfilariae counting techniques using counting chamber, standard slide and membrane (nuclepore) filtration. Annals of Tropical Medicine and Parasitology 73, 437-464.

McMahon, J.E., Magayuka, S.A., Kolstrup, N., Mosha, F.W., Bushrod, F.M., Abaru, D.E. \& Bryan, J.H. (1981) Studies on the transmission and prevalence of bancroftian filariasis in four villages of Tanzania. Annals of Tropical Medicine and Parasitology 75, 415-431

Meyrowitsch, D.W., Simonsen, P.E. \& Makunde, W.H. (1995) Bancroftian filariasis: analysis of infection and disease in five endemic communities of northeastern Tanzania. Annals of Tropical Medicine and Parasitology 89, 653-663.

Muhondwa, E.P.Y. (1978) The Human Behavioral and Social Economical Aspects of Bancroftian Filariasis among the Rural Coastal Population of Tanga, Tanzania. MSc. Dissertation. University of Nottingham, UK.
Olszewski, W.L., Jamal, S., Manokaran, G., Pani, S., Kumaraswami, V., Kubicka, U., Lukomska, B., Dworczynskia, S.E. \& Meisel-Mikolajczyk, F. (1997) Bacteriologic studies of skin, tissue fluid, and lymph nodes in patients with filarial lymphoedema. American Journal of Tropical Medicine and Hygiene 57, 7-15.

Ottesen, E.A., Duke, B.O., Karam, M. \& Behbehani, K. (1997) Strategies and tools for the control/ elimination of lymphatic filariasis. Bulletin of the World Health Organization 75, 491-503.

Punkosdy, G.A., Addiss, D.G. \& Lammie, P.J. (2003) Characterization of antibody responses to Wolabachia surface protein in humans with lymphatic filariasis. Infection and Immunology 71, 5104-5114.

Shenoy, R.K., Sandhya, K., Suma, T.K. \& Kumaraswami, V. (1995) A preliminary study of filariasis related acute adenolymphangitis with special reference to precipitating factors and treatment modalities. South East Asian Journal of Tropical Medicine and Public Health 26, 301-305.

Stadnyk, A.W. \& Gauldie, J. (1991) The acute phase protein response during parasitic infection. Immunology Today 12, A7-A12.

Turner, P.F., Kirk, A., Rockett, K.A., Ottesen, E.A., Francis, H., Awadzi, K. \& Clark, I.A. (1994) Interleukine- 6 and tumour necrosis factor in the pathogenesis of adverse reactions after treatment of lymphatic filariasis and onchocerciasis. Journal of Infectious Diseases 169, 1071-1075.

Titus, R.G., Barbara, S. \& Ceremi, A. (1991) The involvement of TNF, IL-1 and IL-6 in the immune response to protozoan parasites. Immunology Today, 12, A13-A16.

Wegesa, P., McMahon, J.E., Abaru, D.E. Hamilton, P.J.S., Marshall, T.F.de C. \& Vaughan, J.P. (1979) Tanzania Filariasis Project. Survey methodology and clinical manifestations of bancroftian filariasis. Acta Tropica 36, 369377.

Yazdnbakhsh, M., Aarden, L. \& Portono, F. (1992) Serum interleukin-6 levels and adverse reactions to diethylcarbamazine in lymphatic filaraisis. Journal of Infectious Diseases 166, 453-454. 